\title{
ECOLOGÍA URBANA DE AVES: RELACIÓN DE LAS PLANTAS, CLIMA Y RUIDO CON LA BIODIVERSIDAD DE AVES EN LA CIUDAD DE IQUITOS, PERÚ
}

\author{
Natalia C. ANGULO PEREZ1, José A. ARMAS SILVA, Ricardo ZÁRATE GÓMEZ² y Pedro E. PÉREZ- \\ PEÑA $^{3,4}$ \\ 1 Universidad Nacional de la Amazonía Peruana. Facultad de Ciencias Biológicas. Calle Pevas quinta cuadra. \\ nataliaangul02797@gmail.com \\ 2. Instituto de Investigaciones de la Amazonía Peruana (IIAP). Programa de Investigación en Cambio Climático, Desarrollo \\ Territorial y Ambiente (PROTERRA). \\ 3. Instituto de Investigaciones de la Amazonía Peruana (IIAP). Programa de Investigación de Biodiversidad Amazónica \\ (PIBA). \\ 4. Yavarí: Conservación y Uso Sostenible (YAVACUS), Perú.
}

\begin{abstract}
RESUMEN
La ecología urbana brinda directrices a las políticas de desarrollo urbano, para que las sociedades humanas vivan en ambientes más saludables y naturales. Las aves son buenas indicadoras de los ecosistemas naturales, por tal razón con el objetivo de determinar la relación de las plantas, clima y ruido con la biodiversidad de aves urbanas de la ciudad de Iquitos (Perú), se utilizó el método de conteo por puntos en seis de las principales plazas de la ciudad. El diseño de muestreo en cada plaza consistió de cuatro puntos de observación, y se colectó información durante tres días consecutivos, desde las 6:00 a 9:00 h y 16:00 a 18:00 h. La riqueza de aves fue de 50 especies, con mayor abundancia de Columba livia, Coragyps atratus y Tyrannus savana, y la riqueza de plantas fue de 19 especies, con dominancia de Adonidia merrillii, Elaeis oleifera, Livistona chinensis y Ficus benjamina. No hubo diferencias en la temperatura ambiental entre las plazas de la ciudad de Iquitos, pero si hubo diferencias en la temperatura del suelo, humedad relativa y ruido. Se observó la existencia de una relación entre el índice de abundancia de aves carnívoras con la riqueza de especies de plantas en general, especies de plantas nativas árboles. La presencia de plantas, sobre todo de árboles de altos proporcionaría mayor oferta de alimento, sitios de perchado y de nidificación a las especies de aves carnívoras. Finalmente, se concluye que, a un mayor número de especies de aves carnívoras en un ambiente urbano mayor será el avance hacia una ciudad verde.
\end{abstract}

PALABRAS CLAVE: Aves urbanas, Ciudad de Iquitos, Clima, Plantas, Ruido.

\section{URBAN BIRD ECOLOGY: RELATIONSHIP OF THE PLANTS, WEATHER AND NOISE WITH THE BIRD BIODIVERSITY IN THE IQUITOS CITY, PERU.}

\begin{abstract}
Urban ecology provides guidelines for urban development policies, so that human societies live in healthier and more natural environments. Birds are good indicators of natural ecosystems, for this reason with the aim of determining the relationship of plants, climate and noise with the biodiversity of urban birds in the city of Iquitos (Peru), the method of counting by points was used in six of the main squares of the city. The sampling design in each square consisted of four observation points, and information was collected during three consecutive days, from 6:00 a.m. to 9:00 h and 16:00 to 18:00 h. The richness of birds was of 50 species, with greater abundance of Columba livia, Coragyps atratus and Tyrannus savana, and the plant richness was of 19 species, with dominance of Adonidia merrillii, Elaeis oleifera, Livistona chinensis and Ficus benjamina. The environmental temperature between the squares do not differ, but there was in the soil temperature, relative humidity and noise. There is a relationship between the abundance index of carnivorous birds and the richness of plantspecies in general, native plants and richness of trees species. The presence of plants, especially tall trees, would provide a greater supply of food, perching and nesting sites for carnivorous bird species. The greater the number of carnivorous species in an urban environment, the greater the advance towards a green city.
\end{abstract}

KEYWORDS: Iquitos city, Noise, Plants, Urban Birds, Weather. 


\section{INTRODUCCIÓN}

Las ciudades han crecido de manera muy rápida a expensas de espacios que anteriormente eran naturales, sin tener un plan demográfico que indique hacia donde crecer (Ochoa \& Pasquali, 2010). Este fenómeno denominado urbanización ejerce presión sobre aquellas especies silvestres cuyo hábitat está siendo alterado, por ejemplo, las especies de aves (Ochoa \& Pasquali, 2010).

La biodiversidad de aves en un determinado lugar está estrechamente relacionada con la condición de su hábitat, muchas de ellas son sensibles a ligeros cambios (Navarro-Sigüenza et al., 2014).

El conocimiento de las aves que resguardan las áreas verdes de la ciudad, es de vital importancia para definir posibles propuestas de lineamientos para la planificación urbana, que mantengan, restauren y/o mejoren la diversidad biológica de las ciudades o para la estructuración de programas de control de calidad ambiental. La importancia del estudio de aves radica también en que son consideradas como indicadores biológicos muy eficientes que, con su presencia o ausencia, indican la calidad ambiental en áreas naturales o urbanas (Londoño-Betancourth, 2013).

Hay muchos estudios de la ornitofauna en los bosques de la Amazonía noreste peruana (Salazar et al., 2003; Álvarez et al., 2012; Haven et al., 2015), sin embargo, los estudios en ambientes urbanos son limitados. Un estudio de aves en ambientes urbanos y periurbanos de la ciudad de Iquitos y bosque de Varillal determinó que la diversidad en las tres zonas es alta con dominancia de algunas especies. El ambiente urbano con 56 especies estuvo dominado por especies del orden Passeriformes, mientras que el ambiente periurbano con 84 especies estuvo dominado por los órdenes Passeriformes, Psittaciformes y Piciformes (Orbe \& Quispe, 2015).

En la región costa, se realizaron diversos estudios de aves urbanas, es así que, en la ciudad de Lima, en el campus de la Universidad Nacional Agraria La Molina (UNALM) se registraron 46 especies de aves, la mayoría de ellas del orden Passeriformes (Takano \& Castro, 2007); años después, el estudio fue actualizado y se registraron 48 especies de aves en el mismo campus (Castillo et al., 2014). Un estudio de aves silvestres y su correlación con la cobertura vegetal de parques y jardines determinó que la cobertura vegetal es una de las variables determinantes en la composición y estructura de la avifauna (Nolazco, 2012).

El tamaño del parque y la cobertura vegetal presente pueden influenciar en la riqueza y diversidad de aves (Berget, 2006), aunque el ruido es el factor que puede influenciar más en la diversidad de aves; existen diversas tolerancias al ruido dependiendo de la especie (Romero, 2015).

En el Perú, son pocos los trabajos realizados en ecología y ornitología urbana (Castillo et al., 2014), la mayor parte de los estudios de ecología urbana han sido realizados en la región de la costa, particularmente en el área metropolitana de Lima y en la ciudad de Piura (Castañeda et al., 2013), existiendo desconocimiento en la región de la sierra y selva de Perú. Este desconocimiento, dificulta crear planes de ordenamiento urbano en las principales ciudades amazónicas.

En este sentido, el estudio se realizó con la finalidad de describir la diversidad de la ornitofauna urbana y evaluar si es influenciada por las plantas, temperatura, humedad y ruido en la ciudad de Iquitos. Esta información será muy importante para la recuperación de espacios verdes en centros urbanos, promoviendo una política de conservación de áreas verdes para las aves y otras especies de fauna que en la planificación del espacio público raramente son consideradas. Además, el estudio servirá a los diferentes municipios como insumo principal para su gestión y planeamiento de una ciudad verde.

\section{MATERIALES Y MÉTODOS}

\section{ÁREA DE ESTUDIO}

\section{Ubicación}

El presente estudio se realizó en seis plazas de la ciudad de Iquitos que son: 28 de Julio $\left(3^{\circ} 45^{\prime} 16.47^{\prime \prime}\right.$

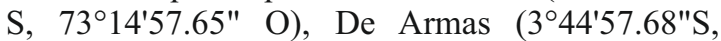
$\left.73^{\circ} 14^{\prime} 39.81^{\prime \prime O}\right)$, Sargento Lores $\left(3^{\circ} 44^{\prime} 52.26^{\prime \prime S}\right.$, $\left.73^{\circ} 15^{\prime} 8.15^{\prime \prime O}\right)$, Almirante Miguel Grau $\left(3^{\circ} 43^{\prime} 42.67^{\prime \prime S}, 73^{\circ} 14^{\prime} 39.77^{\prime \prime O}\right)$, Francisco Bolognesi $\left(3^{\circ} 45^{\prime} 52.29^{\prime \prime} \mathrm{S}, 73^{\circ} 15^{\prime} 23.71^{\prime \prime O}\right)$ y José Abelardo Quiñones o Plaza Roja $\left(3^{\circ} 46^{\prime} 30.18^{\prime \prime S}\right.$, $\left.73^{\circ} 17^{\prime} 22.17^{\prime \prime} \mathrm{O}\right)$, en el departamento de Loreto, Perú (Figura 1). La ciudad de Iquitos se encuentra ubicada en la selva baja, sobre la margen izquierda del río Itaya, al noreste de la Amazonía Peruana. La ciudad limita por el norte y noroeste con el río Nanay, y por el este y sur con el río Itaya.

El clima es tropical cálido, húmedo y lluvioso, con temperaturas mínimas medias de $20-22{ }^{\circ} \mathrm{C}$ y máximas entre $29-31{ }^{\circ} \mathrm{C}$, las medias anuales superan los $25^{\circ} \mathrm{C}$ y las máximas absolutas en Iquitos no sobrepasan de $35^{\circ} \mathrm{C}$. Las precipitaciones anuales son superiores a 2000 milímetros y localmente pueden superar los 5000 milímetros. La humedad relativa media anual en la región es bastante alta, con valores que oscilan entre 80 y $90 \%$ durante todo el 


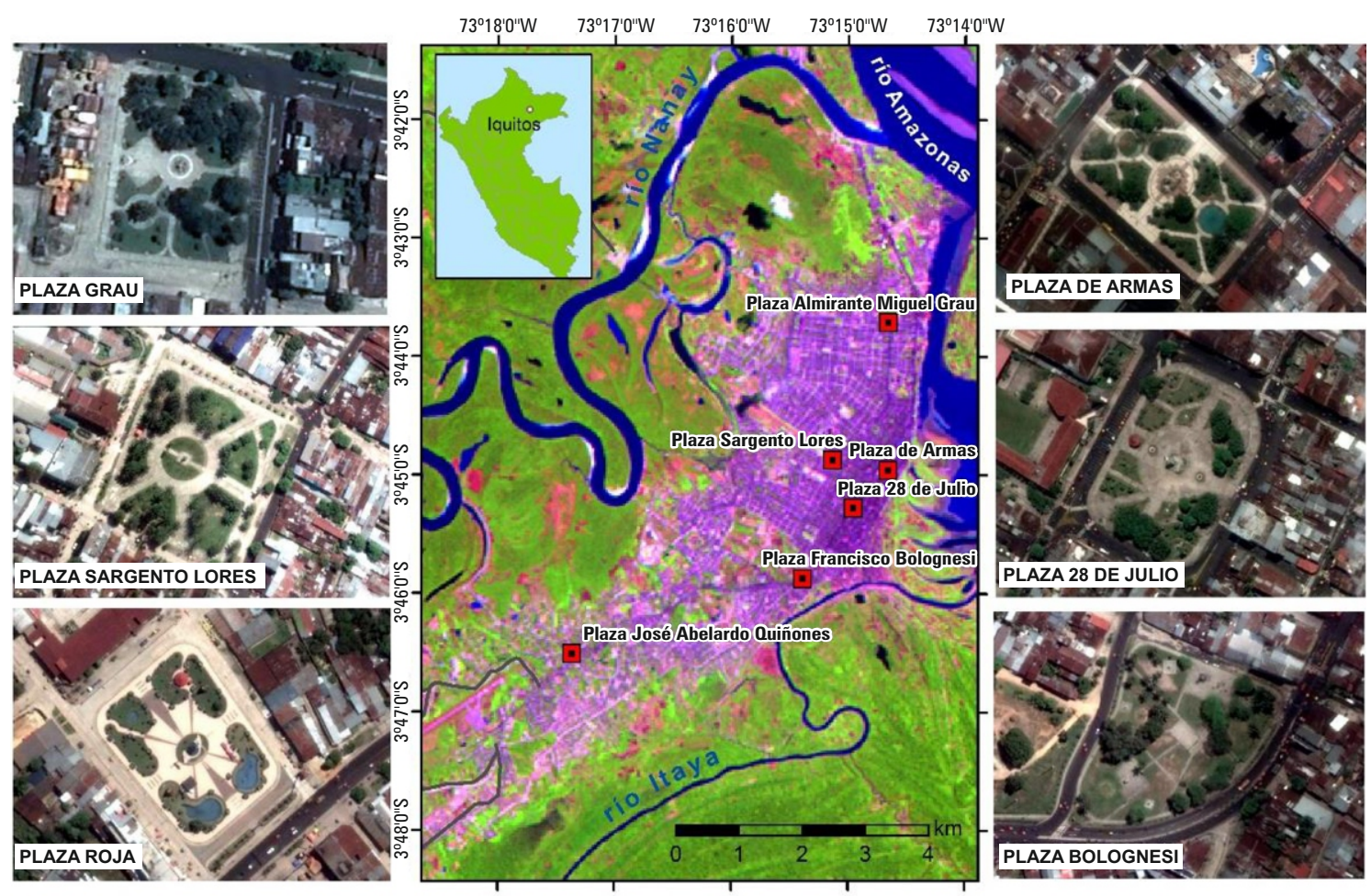

Figura 1. Mapa de ubicación de las seis plazas estudiadas en la ciudad de lquitos.

año. La humedad atmosférica es alta a lo largo de todo el año, favorecida por la evaporación de los abundantes cursos de agua y zonas pantanosas, así como la evapotranspiración del bosque (Dourojeanni, 2013; Marengo, 1998). Entre los meses de enero a junio se da la temporada de aguas altas o creciente, y entre los meses de julio a diciembre, la temporada de vaciante.

\section{Flora}

En la ciudad de Iquitos se pueden encontrar diferentes grupos de plantas, principalmente aquellas que han sido cultivadas por el hombre. La ornamentación de la ciudad ha sido materia de crítica desde hace mucho tiempo debido a que muchas especies han sido introducidas desde varios lugares del mundo; por ejemplo, Ficus benjamina conocido comúnmente como "ficus" es una especie nativa de Asia (Cárdenas et al., 2011) que fue introducida en la década de los 80 's y que representa aproximadamente el $13.62 \%$ de las plantas ornamentales presentes en la ciudad de Iquitos (Zárate \& Mori, 2012).

Entre las especies de plantas más destacadas de la ciudad de Iquitos se encuentran: Mangifera indica "mango", Cocus nucifera "coco", Elaeis oleifera "palma aceitera", Euterpe precatoria "huasaí", Mauritia flexuosa "aguaje", Carica papaya "papaya", Couepia subcordata "parinari", Terminalia catappa "castañilla", Hibiscus rosasinensis "cucarda", Ficus benjamina "ficus", Syzygium cumini "aceituna dulce", Syzygium malaccense "pomarosa", Pouteria caimito “caimito", entre otras (Zárate \& Mori, 2012).

\section{METODOLOGÍA}

\section{Ornitofauna}

Los muestreos de la ornitofauna de las principales plazas de la ciudad de Iquitos se realizaron del 14 de marzo al 27 de abril del 2017. Cada una de las seis plazas fueron muestreadas durante tres días consecutivos, entre las 6:00 a 9:00 horas y 16:00 a 18:00 horas, con un total de 90 horas hombre. El área de las plazas fue medida usando la imagen más actual de Google Earth y el programa ArcGIS.

El método de muestreo fue el de conteo por puntos, para ello cada plaza fue dividida en cuatro cuadrantes. Los puntos de conteo se ubicaron en el centro de cada cuadrante y se muestreó durante diez minutos. En una ficha de colecta, se anotaron el 
número de individuos por especie, microhábitat, comportamiento y nombre del punto, además se realizó un registro fotográfico de la mayoría de las especies. También se colectó información de temperatura ambiental, de suelo y humedad relativa. La identificación de las especies de aves se realizó según las consideraciones descritas y reportada por Schulenberg et al. (2010) y Plenge (2017).

Los ítems alimenticios utilizados en este estudio fueron los siguientes: carnívoro, carroñero, frugívoro, frugívoro - insectívoro, granívoro, granívoro - frugívoro, insectívoro, nectarívoro, omnívoro y piscívoro (Velásquez-Valencia et al., 2003; Crocce, 2011; Strewe et al., 2009; Velásquez, 2009; Bazán, 2012; Castro, 2012; Nolazco, 2012; Castro \& Gonzales, 2014; Siqueira et al., 2015; Peña-Núñez \& Claros-Morales, 2016).

\section{Plantas}

El estudio consideró tres tipos de plantas que fueron: arbusto, palmera y árbol. Se midió el diámetro de las plantas, la altura y cobertura. Para la herborización se utilizó lo propuesto por Judd et al., (1999). La identificación de las especies de plantas fue realizada usando la clave de Vásquez (1997); también utilizamos las fotografías de las exicatas del Missouri Botanical Garden (http://www. tropicos.org/) y del Field Museum of Natural History (https://www.fieldmuseum.org/). Para los estados actuales de los nombres utilizamos: The Plant List (http://www.theplantlist.org), mientras que, para el sistema de clasificación de las familias empleado fue según lo propuesto por el APG III (2009). Las muestras botánicas se colectaron con los permisos otorgados por la Municipalidad Provincial de Maynas y el permiso de investigación con colecta $\mathrm{N}^{\circ} 0068$-2015-SERFOR-DGGSPFFS otorgado por el Servicio Forestal y de Fauna Silvestre (SERFOR) del Ministerio de Agricultura, y fueron depositados en el Herbario Iquitos (HIQ) del IIAP y el Museo Amazonense (AMAZ) de la Universidad Nacional de la Amazonía Peruana (UNAP).

\section{Clima}

En cada punto de conteo establecido, se midieron parámetros como temperatura ambiental, temperatura del suelo y humedad relativa al inicio y final del muestreo. La temperatura y humedad ambiental fueron medidas con un termohigrómetro, mientras que la temperatura del suelo fue medida con un termómetro adaptado al suelo.

\section{Ruido}

El ruido se midió con el smartphone Motorola
3G, 2014 usando la aplicación Noise meter. Esta aplicación brinda resultados confiables del ruido sólo para fines comparativos de investigación, pero no para detectar límites legales (Kardous \& Shaw, 2014). El registro del ruido se realizó durante un minuto en cada uno de los puntos de conteo establecidos y en la hora de muestreo de la ornitofauna.

\section{ANÁLISIS DEDATOS \\ Riqueza, dominancia y abundancia de aves y plantas}

La riqueza de aves se analizó en base al número de especies observadas y esperadas. Se calculó el número de especies esperadas con los índices de Chao 1, Chao 2, Chao \& Lee 1, Chao \& Lee 2, Jackknife 1, Jackknife 2, Bootstrap y Michaelis Menten. La dominancia en la riqueza de plantas fue estudiada mediante el índice de Simpson y gráficos de curva de Whittaker, donde el número cercano a cero, indicó mayor dominancia.

Se consideró al índice de abundancia como un estimador de la población o abundancia de aves, para ello el número de individuos se dividió entre el esfuerzo (horas de muestreo). En el caso de las plantas se estimó la densidad, el cual es el número total de individuos presentes sobre el área total de la plaza.

\section{Cobertura de plantas}

Para estimar la cobertura de las plantas en las plazas se tomaron dos medidas de la copa de cada planta, desde un extremo a otro de forma diagonal. Para estimar el área total de la copa del árbol, se utilizó la fórmula del área de un círculo $\left(\pi \times \mathrm{r}^{2}\right)$, donde el radio se obtuvo del promedio de las dos medidas y el $\pi$ es una constante de 3.1416. Finalmente, se realizó la sumatoria de las áreas de las copas presentes en cada zona de estudio para obtener el resultado total y transformarlo a porcentaje.

\section{Descripción del clima y el ruido}

Para describir los factores climáticos y los patrones de ruido de las plazas estudiadas se empleó la estadística descriptiva tales como la mediana (medida de tendencia central), rango intercuartílico y rangos (medida de precisión), además de valores atípicos, todo ello resumido en un gráfico de cajas o boxplot. Las comparaciones de la temperatura, humedad y ruido entre plazas fueron realizadas con la prueba Kruskal Wallis por tener diferentes tipos de distribuciones. Estos cálculos fueron realizados con el programa Sigmaplot 11.0. 
Para el caso del ruido se empleó el factor de corrección para homogenizar los datos obtenidos, y evitar variaciones por tipo de celular y micrófono (Kardous \& Shaw 2014). La homogenización consistió en sumar 6.2 al valor medio del ruido. Esta constante fue obtenida mediante comparaciones del celular control (celular nuevo con mejor micrófono) con el resto de celulares.

Las unidades fueron expresadas en Leq (nivel sonoro equivalente), por ser más estable.

\section{Identificación de la causa de la biodiversidad}

Para identificar las variables (flora, clima y ruido) más importantes que explican la mayor variabilidad en los patrones de biodiversidad de aves en las plazas, se utilizó el Análisis de Componentes Principales mediante el uso de una matriz de correlación. Ello, por tratarse de un análisis con diferentes unidades de medida, se utilizó el programa Community Analysis Package (CAP) 4.0.

\section{RESULTADOS}

\section{RIQUEZA, ABUNDANCIA Y DOMINANCIA DE AVES}

Los muestreos realizados en las seis plazas principales de Iquitos lograron registrar 26,530 avistamientos de 50 especies de aves, distribuidas en
10 órdenes, 14 familias y 44 géneros. La riqueza esperada fue de 55 especies de acuerdo a la aplicación de los índices paramétricos y no paramétricos, es decir, se logró registrar el $90.9 \%$ del total de especies (Figura 2).

A nivel global, las aves más abundantes presentes en las plazas de la ciudad de Iquitos fueron Columba livia (31.36 ind./hora), Coragyps atratus (23.14 ind./hora) y Tyrannus savana (5.79 ind./hora). Las especies de aves con mayor abundancia en la Plaza 28 de Julio, Sargento Lores, Grau y Bolognesi fueron Coragyps atratus y Columba livia, y en la Plaza Quiñones las más abundantes fueron Columba livia y Progne chalybea (Tabla 1).

La Plaza 28 de Julio, con 0.53 , presenta la más alta dominancia respecto a las demás plazas en estudio (Tabla 2), debido a que $C$. livia es desproporcionadamente la más dominante sobre las demás especies (1,384 avistamientos), seguido por C. atratus (307) y $P$. chalybea (95), mientras más registros de una especie, mayor será la dominancia. La dominancia por ítems alimenticios, mostró que tanto la Plaza 28 de Julio como la Plaza de Armas, tuvieron dominancia de granívoras - frugívoras; mientras que, en la Plaza Sargento Lores, Grau y Bolognesi fueron las carroñeras, y en la Plaza Quiñones, fueron las insectívoras (Figura 3).

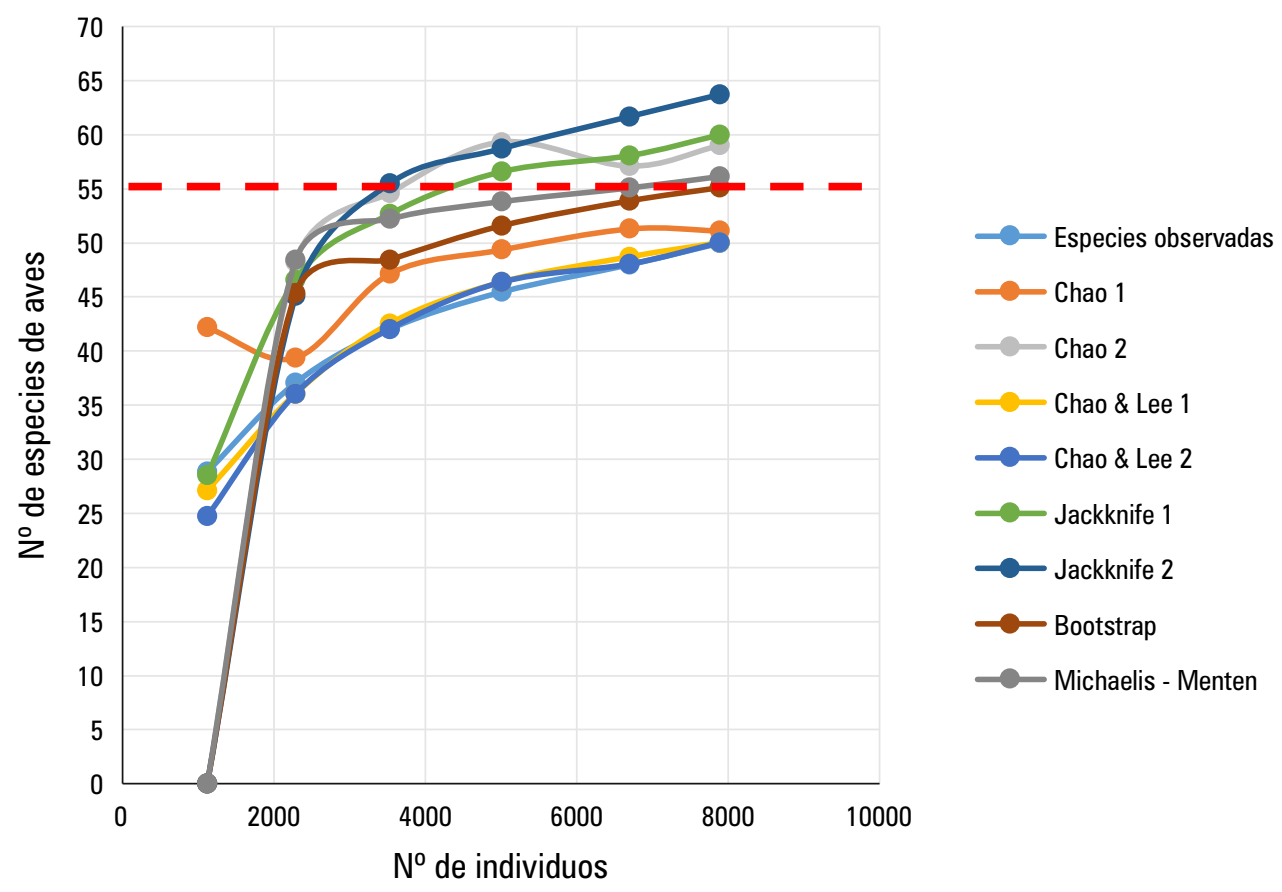

Figura 2. Riqueza observada y esperada de especies de aves en las plazas de lquitos, según estimadores paramétricos y no paramétricos. La línea roja interrumpida indica la riqueza esperada. 
Tabla 1. Abundancia de especies de aves en las plazas de la ciudad de lquitos según su densidad poblacional. Cr: Carnívoro, Cñ: Carroñero, Fr: Frugívoro, Fr-In: Frugívoro-Insectívoro, Gr: Granívoro, Gr-Fr: Granívoro-Frugívoro, In: Insectívoro, Nc: Nectarívoro, Om: Omnívoro, Ps: Piscívoro.

\begin{tabular}{|c|c|c|c|c|c|c|c|c|}
\hline \multirow[b]{2}{*}{ Nombre científico } & \multirow{2}{*}{$\begin{array}{c}\text { Item } \\
\text { alimenticio }\end{array}$} & \multicolumn{7}{|c|}{ Índice de Abundancia (individuos/hora) } \\
\hline & & $\begin{array}{l}28 \text { de } \\
\text { Julio }\end{array}$ & Armas & $\begin{array}{c}\text { Sargento } \\
\text { Lores }\end{array}$ & Grau & Bolognesi & Quiñones & $\begin{array}{c}\text { Total } \\
\text { (Promedio) }\end{array}$ \\
\hline \multicolumn{9}{|l|}{ Accipitridae } \\
\hline Buteo albonotatus & $\mathrm{Cr}$ & 0.07 & 0.00 & 0.07 & 0.07 & 0.00 & 0.07 & 0.04 \\
\hline Rupornis magnirostris & $\mathrm{Cr}$ & 0.13 & 0.53 & 0.07 & 0.07 & 0.20 & 0.00 & 0.17 \\
\hline \multicolumn{9}{|l|}{ Alcedinidae } \\
\hline Megaceryle torquata & Ps & 0.00 & 0.00 & 0.00 & 0.00 & 0.00 & 0.27 & 0.04 \\
\hline \multicolumn{9}{|l|}{ Apodidae } \\
\hline Chaetura brachyura & In & 0.47 & 0.00 & 2.13 & 0.00 & 0.67 & 1.47 & 0.79 \\
\hline Tachornis squamata & In & 0.00 & 0.00 & 0.00 & 0.00 & 0.13 & 1.80 & 0.32 \\
\hline \multicolumn{9}{|l|}{ Ardeide } \\
\hline Ardea alba & Ps & 0.40 & 0.20 & 0.40 & 1.20 & 0.33 & 0.60 & 0.52 \\
\hline Butorides striata & Ps & 0.00 & 0.00 & 0.00 & 0.00 & 0.20 & 0.67 & 0.14 \\
\hline Egretta thula & Ps & 0.00 & 0.00 & 0.00 & 0.07 & 0.00 & 0.00 & 0.01 \\
\hline \multicolumn{9}{|l|}{ Cathartidae } \\
\hline Cathartes aura & Cñ & 0.93 & 1.07 & 0.67 & 0.33 & 0.33 & 1.00 & 0.72 \\
\hline Coragyps atratus & Cñ & 20.47 & 16.60 & 38.27 & 40.07 & 18.20 & 5.27 & 23.14 \\
\hline \multicolumn{9}{|l|}{ Columbidae } \\
\hline Columba livia & Gr-Fr & 92.27 & 28.60 & 21.80 & 15.13 & 8.73 & 21.60 & 31.36 \\
\hline Columbina talpacoti & $\mathrm{Gr}-\mathrm{Fr}$ & 0.53 & 2.73 & 0.33 & 0.67 & 2.27 & 0.07 & 1.10 \\
\hline \multicolumn{9}{|l|}{ Cuculidae } \\
\hline Crotophaga ani & $\ln$ & 0.80 & 1.13 & 0.00 & 0.00 & 0.00 & 1.27 & 0.53 \\
\hline \multicolumn{9}{|l|}{ Emberizidae } \\
\hline Ammodramus aurifrons & $\mathrm{Gr}$ & 0.93 & 0.00 & 0.07 & 0.00 & 0.67 & 0.20 & 0.31 \\
\hline \multicolumn{9}{|l|}{ Falconidae } \\
\hline Falco peregrinus & $\mathrm{Cr}$ & 0.00 & 0.20 & 0.00 & 0.00 & 0.00 & 0.00 & 0.03 \\
\hline Milvago chimachima & $\mathrm{Cr}$ & 0.80 & 0.27 & 0.33 & 0.27 & 0.20 & 0.13 & 0.33 \\
\hline \multicolumn{9}{|l|}{ Fringillidae } \\
\hline Euphonia chlorotica & $\mathrm{Fr}$ & 0.87 & 0.20 & 1.07 & 0.33 & 0.27 & 1.60 & 0.72 \\
\hline \multicolumn{9}{|l|}{ Hirundinidae } \\
\hline Hirundo rustica & In & 0.00 & 0.00 & 0.00 & 2.27 & 0.73 & 0.60 & 0.60 \\
\hline Progne chalybea & $\ln$ & 6.33 & 2.67 & 0.87 & 0.00 & 0.20 & 8.33 & 3.07 \\
\hline \multicolumn{9}{|l|}{ Icteridae } \\
\hline Cacicus cela & $\mathrm{Fr}$ & 0.20 & 0.87 & 0.13 & 0.07 & 0.47 & 0.20 & 0.32 \\
\hline Psarocolius angustifrons & In & 0.00 & 0.07 & 0.13 & 0.73 & 7.07 & 1.80 & 1.63 \\
\hline \multicolumn{9}{|l|}{ Laridae } \\
\hline Phaetusa simplex & Ps & 0.07 & 0.00 & 0.00 & 0.00 & 0.07 & 0.00 & 0.02 \\
\hline \multicolumn{9}{|l|}{ Phalacrocoracidae } \\
\hline Phalacrocorax brasilianus & Ps & 0.00 & 0.07 & 0.00 & 0.00 & 0.00 & 0.00 & 0.01 \\
\hline \multicolumn{9}{|l|}{ Psittacidae } \\
\hline Aratinga weddellii & $\mathrm{Fr}$ & 0.00 & 0.00 & 0.00 & 0.27 & 0.00 & 0.40 & 0.11 \\
\hline
\end{tabular}




\begin{tabular}{|c|c|c|c|c|c|c|c|c|}
\hline \multirow[b]{2}{*}{ Nombre científico } & \multirow{2}{*}{$\begin{array}{c}\text { Item } \\
\text { alimenticio }\end{array}$} & \multicolumn{7}{|c|}{ Índice de Abundancia (individuos/hora) } \\
\hline & & $\begin{array}{l}28 \text { de } \\
\text { Julio }\end{array}$ & Armas & $\begin{array}{c}\text { Sargento } \\
\text { Lores }\end{array}$ & Grau & Bolognesi & Quiñones & $\begin{array}{c}\text { Total } \\
\text { (Promedio) }\end{array}$ \\
\hline Brotogeris cyanoptera & $\mathrm{Fr}$ & 0.00 & 0.00 & 0.00 & 0.00 & 1.33 & 0.00 & 0.22 \\
\hline Brotogeris sanctithomae & $\mathrm{Fr}$ & 0.00 & 0.00 & 0.00 & 0.00 & 3.53 & 0.00 & 0.59 \\
\hline Brotogeris sp. & $\mathrm{Fr}$ & 0.00 & 0.00 & 0.00 & 0.00 & 0.00 & 0.93 & 0.16 \\
\hline Brotogeris versicolurus & $\mathrm{Fr}$ & 0.80 & 0.00 & 0.00 & 1.27 & 3.80 & 0.00 & 0.98 \\
\hline Forpus xanthopterygius & $\mathrm{Fr}$ & 0.00 & 0.00 & 0.07 & 0.00 & 0.00 & 0.00 & 0.01 \\
\hline Psittacara leucophthalmus & $\mathrm{Fr}$ & 1.73 & 1.07 & 9.53 & 0.33 & 3.13 & 0.13 & 2.66 \\
\hline \multicolumn{9}{|l|}{ Thraupidae } \\
\hline Coereba flaveola & Nc & 0.00 & 0.07 & 0.00 & 0.33 & 0.00 & 0.00 & 0.07 \\
\hline Paroaria gularis & $\mathrm{Gr}$ & 0.07 & 0.47 & 0.00 & 0.13 & 0.00 & 0.00 & 0.11 \\
\hline Ramphocelus carbo & Fr-ln & 0.00 & 0.00 & 0.00 & 0.13 & 0.00 & 0.00 & 0.02 \\
\hline Sporophila castaneiventris & $\mathrm{Gr}$ & 0.07 & 0.13 & 0.00 & 0.00 & 0.00 & 0.20 & 0.07 \\
\hline Thlypopsis sordida & $0 \mathrm{~m}$ & 0.07 & 0.00 & 0.07 & 0.27 & 0.07 & 0.27 & 0.12 \\
\hline Thraupis episcopus & Fr-ln & 3.73 & 1.53 & 1.80 & 4.27 & 5.53 & 0.87 & 2.96 \\
\hline Thraupis palmarum & Fr-ln & 0.07 & 2.80 & 0.33 & 0.73 & 1.13 & 0.33 & 0.90 \\
\hline \multicolumn{9}{|l|}{ Trochilidae } \\
\hline Amazilia fimbriata & Nc & 0.00 & 0.07 & 0.53 & 0.27 & 0.07 & 0.00 & 0.16 \\
\hline \multicolumn{9}{|l|}{ Troglodytidae } \\
\hline Troglodytes aedon & $\ln$ & 0.00 & 0.00 & 0.27 & 0.00 & 0.00 & 0.13 & 0.07 \\
\hline \multicolumn{9}{|l|}{ Turdidae } \\
\hline Turdus ignobilis & Fr-ln & 0.20 & 0.00 & 0.20 & 0.40 & 0.00 & 0.00 & 0.13 \\
\hline \multicolumn{9}{|l|}{ Tyrannidae } \\
\hline Contopus virens & $\ln$ & 0.00 & 0.00 & 0.00 & 0.60 & 0.13 & 0.00 & 0.12 \\
\hline Megarynchus pitangua & In & 0.00 & 0.00 & 0.00 & 0.07 & 0.07 & 0.00 & 0.02 \\
\hline Myiodynastes maculatus & $\ln$ & 0.00 & 0.00 & 0.00 & 0.00 & 0.13 & 0.00 & 0.02 \\
\hline Myiozetetes similis & In & 0.87 & 0.00 & 0.00 & 1.67 & 0.33 & 0.73 & 0.60 \\
\hline Phaeomyias murina & $\ln$ & 0.00 & 0.00 & 0.00 & 0.80 & 0.00 & 0.00 & 0.13 \\
\hline Pitangus sulphuratus & In & 1.33 & 1.87 & 2.27 & 2.00 & 2.93 & 1.73 & 2.02 \\
\hline Todirostrum maculatum & $\ln$ & 0.20 & 0.00 & 0.00 & 0.13 & 0.80 & 0.00 & 0.19 \\
\hline Tyrannus albogularis & $\ln$ & 0.00 & 0.00 & 0.00 & 0.33 & 0.00 & 0.00 & 0.06 \\
\hline Tyrannus melancholicus & $\ln$ & 3.20 & 5.07 & 2.60 & 2.67 & 4.53 & 2.87 & 3.49 \\
\hline Tyrannus savana & In & 1.07 & 16.80 & 12.00 & 1.60 & 0.13 & 3.13 & 5.79 \\
\hline
\end{tabular}

\section{RIQUEZA, ABUNDANCIA Y DOMINANCIA DE PLANTAS}

En las seis plazas principales de Iquitos se registraron 388 individuos y 19 especies de plantas, distribuidas en 9 órdenes, 11 familias y 18 géneros. De las 19 especies, 5 fueron especies nativas, 11 fueron exóticas y 3 especies con origen indeterminado. En cuanto a hábitos, una especie fue arbustiva, siete especies de palmera y 12 especies de árboles.

En este estudio, las plantas más abundantes reportadas en la ciudad de Iquitos fueron Adonidia merrillii (17.18 ind./ha), Elaeis oleifera (14.21 ind./ha), Livistona chinensis (8.32 ind./ha) y Ficus benjamina (6.78 ind./ha). Las especies de plantas con mayor densidad en la Plaza 28 de Julio fueron Dypsis lutescens (17.90 ind./ha) y Ficus benjamina (10.74 ind./ha), en la Plaza de Armas fueron Livistona chinensis (27.78 ind./ha) y Adonidia merrillii (15.28 ind./ha), en la Plaza Sargento Lores fue la palma aceitera Elaeis oleifera (48.56 ind./ha) y, en la Plaza Grau fueron Syzygium cumini (24.87 ind./ha) y Livistona chinensis (17.10 ind./ha), en la Plaza Bolognesi fue solamente la palmera Elaeis 
Tabla 2. Área, cobertura de plantas (\%), dominancia de aves y dominancia de plantas en las plazas de lquitos.

\begin{tabular}{lccccc}
\hline \multicolumn{1}{c}{ Plaza } & $\begin{array}{c}\text { Área de } \\
\text { cobertura de } \\
\text { plantas (ha) }\end{array}$ & $\begin{array}{c}\text { Área de } \\
\text { la plaza (ha) }\end{array}$ & $\begin{array}{c}\text { Porcentaje de } \\
\text { cobertura de } \\
\text { plantas en las } \\
\text { plazas (\%) }\end{array}$ & $\begin{array}{c}\text { Dominancia } \\
\text { de aves }\end{array}$ & $\begin{array}{c}\text { Dominancia } \\
\text { de plantas }\end{array}$ \\
\hline 28 de Julio & 0.34 & 1.40 & 24.62 & 0.53 & 0.76 \\
\hline Armas & 0.22 & 0.72 & 29.98 & 0.80 & 0.80 \\
\hline Sargento Lores & 0.42 & 1.28 & 33.09 & 0.76 & 0.71 \\
Grau & 0.23 & 0.64 & 36.42 & 0.70 & 0.73 \\
Bolognesi & 0.36 & 1.00 & 35.93 & 0.88 & 0.77 \\
\hline Quiñones & 0.03 & 0.70 & 4.52 & 0.82 & 0.07 \\
\hline
\end{tabular}

oleifera (24.99 ind./ha) y en la Plaza Quiñones de las dos especies presentes, la más abundante fue Adonidia merrillii (74.77 ind./ha)(Tabla 3).

La Plaza Quiñones presentó la mayor dominancia con 0.07 (Tabla 2), debido a la dominancia de Adonidia merrillii con 54 individuos. En las plazas 28 de Julio, De Armas, Sargento Lores, Grau y Bolognesi se reportó una menor dominancia porque las especies de plantas tuvieron una distribución regularmente homogénea en cuanto al número de individuos.

\section{COBERTURA DE PLANTAS}

Las seis plazas de la ciudad de Iquitos tuvieron una cobertura de plantas de 1.6 ha $(27.87 \%$, $100 \%=5.74$ ha). La Plaza Grau con $36.42 \%$ de cobertura es la que posee una mayor cobertura de plantas; seguida de la Plaza Bolognesi con 35.93\%. La que tuvo menor cobertura fue la Plaza Quiñones, cuya área cubierta por plantas fue de sólo $4.52 \%$ (Tabla 2).

\section{TEMPERATURA, HUMEDAD Y RUIDO}

Entre las seis plazas de la ciudad de Iquitos, no hubo diferencias significativas en la temperatura ambiental $(\mathrm{H}=5.89, \mathrm{P}=0.317)$. La temperatura ambiental de la Plaza 28 de Julio fue de $27.88^{\circ} \mathrm{C}$ (25.70 - 32.18, rango intercuartílico); en la Plaza de Armas fue de $27.30{ }^{\circ} \mathrm{C}$ (26.10 - 29.10); en la Plaza Sargento Lores fue de $26.63^{\circ} \mathrm{C}$ (25.73 - 30.10); Plaza Grau, $26.80{ }^{\circ} \mathrm{C}$ (25.88 - 28.35); Plaza Bolognesi, $27.53{ }^{\circ} \mathrm{C}(25.85$ - 29.18). Finalmente, en la Plaza Quiñones fue de $26.73^{\circ} \mathrm{C}(26.08$ - 29.30) (Figura 4).

Entre las seis plazas muestreadas existen diferencias significativas en la temperatura del suelo $(\mathrm{H}=30.30, \mathrm{P}<0.001)$. Los valores de la temperatura del suelo en la Plaza Grau y la Plaza Quiñones presentaron valores diferentes a las demás plazas de la ciudad de Iquitos (Tabla 4).

Los mayores valores de temperatura media del suelo fueron en la Plaza Quiñones con $28.13{ }^{\circ} \mathrm{C}$ (26.85 - 30.93, rango intercuartílico), Plaza de Armas con $27.65^{\circ} \mathrm{C}(26.80$ - 29.08) y en la Plaza 28 de Julio con $27.50{ }^{\circ} \mathrm{C}(25.88$ - 30.85). El valor más bajo fue registrado en la Plaza Grau con $26.60{ }^{\circ} \mathrm{C}$ (25.50 - 27.63). Los valores medios fueron registrados en la Plaza Sargento Lores con $26.78^{\circ} \mathrm{C}$ (26.20 - 30.43) y en la Plaza Bolognesi con $27.23^{\circ} \mathrm{C}$ (25.33 - 29.18) (Figura 4).

La humedad relativa registrada entre las plazas fueron diferentes $(\mathrm{H}=50.45, P<0.001)$. Los valores de la humedad relativa en la Plaza Bolognesi y la Plaza Quiñones fueron las que se diferencian significativamente de las demás plazas citadas en este estudio (Tabla 4). Los mayores valores de humedad relativa fueron en la Plaza Quiñones con $77.25 \%$ (62.63 - 82.50, rango intercuartílico), Plaza Bolognesi con 74.74\% (69.50 - 85.13) y en la Plaza 28 de Julio con $70.25 \%$ (66 - 75). El valor más bajo fue registrado en la Plaza Sargento Lores, representando el 66.25\% (62.38 - 71.50). Los valores medios fueron registrados en la Plaza de Armas con 67\% (55.50 - 72.63) y en la Plaza Grau con 68.25\% (60.38 - 75.75) (Figura 4).

El ruido registrado en las plazas en estudio también mostró diferencias significativas entre ellas $(\mathrm{H}=3253.21 .45, P<0.001)$ (Tabla 4). Los mayores valores fueron registrados en la Plaza Bolognesi con $73.70 \mathrm{~dB}$ (72.70 - 74.50, rango intercuartílico); Plaza de Armas, con $73.40 \mathrm{~dB}$ (72.30 - 74.20) y en la Plaza 28 de Julio con $72.40 \mathrm{~dB}$ (71.30 - 74.70). El más bajo fue registrado en la Plaza Sargento Lores con 71.70 dB (69.60 - 72.10). Los valores medios fueron registrados en la Plaza Quiñones con $71.80 \mathrm{~dB}$ (70.80 - 72.80), mientras que en la Plaza Grau fue de $72 \mathrm{~dB}(70.40$ - 73.10) (Figura 4). 
28 de Julio

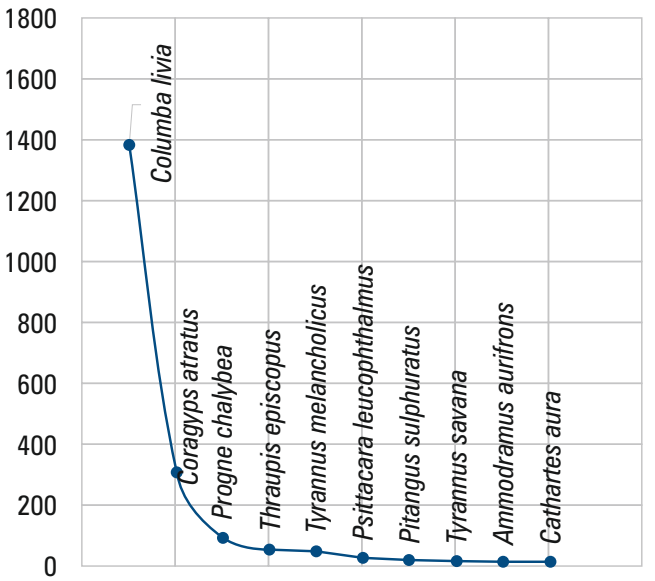

Bolognesi

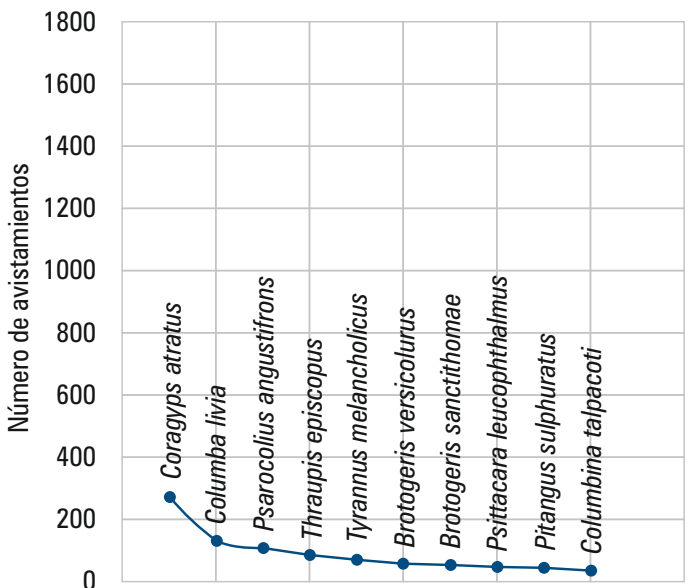

Quiñones

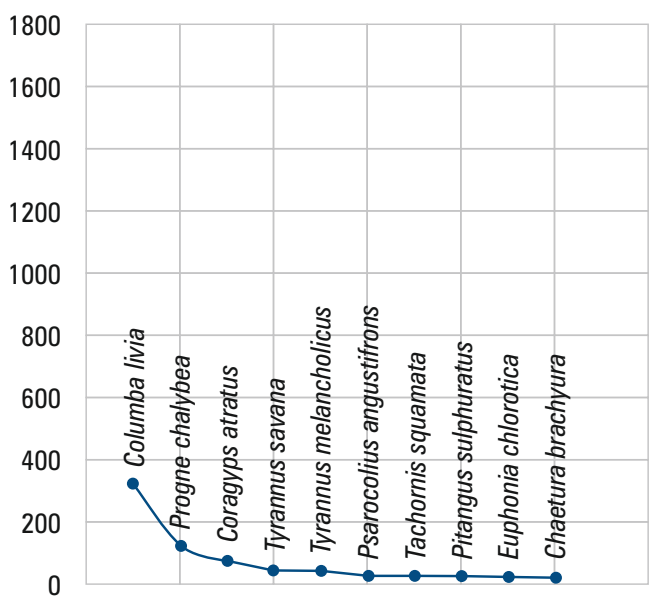

Armas

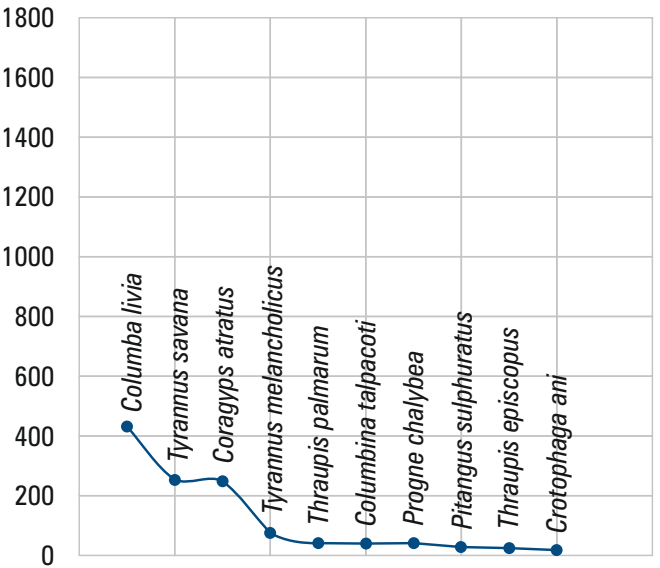

Grau

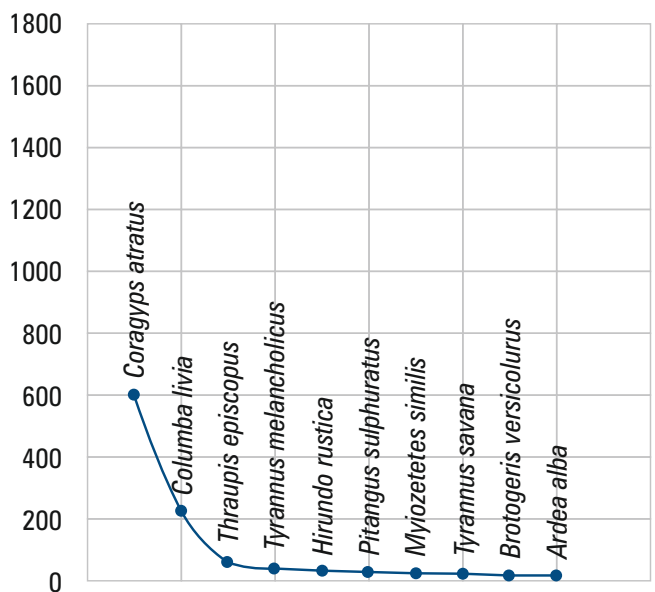

Sargento Lores

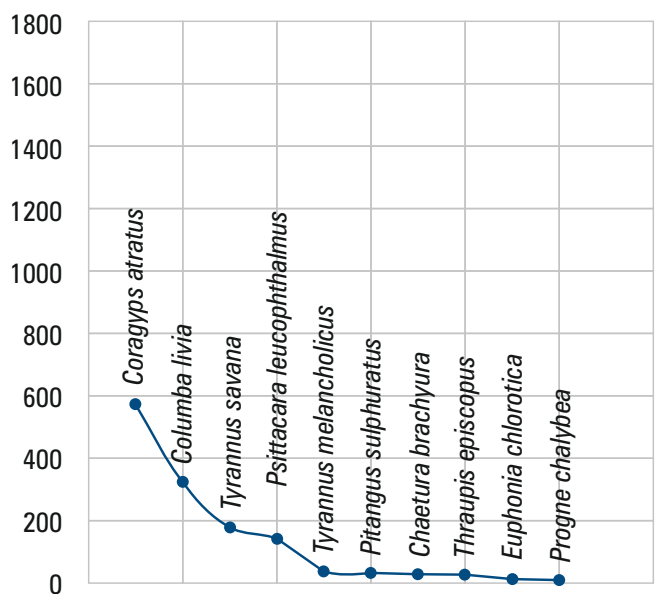

Figura 3. Curvas de Whittaker de las especies de aves registradas en las plazas de lquitos. 
Tabla 3. Densidad de especies de plantas en las plazas de lquitos según su densidad poblacional. Ar: Árbol, Ab: Arbusto, PI: Palmera, Ex: Exótica, Nt: Nativa, Ind: Indeterminado.

\begin{tabular}{|c|c|c|c|c|c|c|c|c|c|}
\hline \multirow[b]{2}{*}{ Nombre científico } & \multirow[b]{2}{*}{ Hábito } & \multirow[b]{2}{*}{ Origen } & \multicolumn{7}{|c|}{ Densidad (Ind./ha) } \\
\hline & & & $\begin{array}{l}28 \text { de } \\
\text { Julio }\end{array}$ & Armas & $\begin{array}{c}\text { Sargento } \\
\text { Lores }\end{array}$ & Grau & Bolognesi & Quiñones & Total \\
\hline \multicolumn{10}{|l|}{ Anacardiaceae } \\
\hline Mangifera indica & $\mathrm{Ar}$ & Ex & 0.00 & 0.00 & 1.57 & 0.00 & 0.00 & 0.00 & 0.26 \\
\hline \multicolumn{10}{|l|}{ Annonaceae } \\
\hline Annona montana & $\operatorname{Ar}$ & Ex & 0.72 & 0.00 & 0.00 & 0.00 & 0.00 & 0.00 & 0.12 \\
\hline \multicolumn{10}{|l|}{ Arecaceae } \\
\hline Adonidia merrillii & $\mathrm{PI}$ & Ex & 5.01 & 15.28 & 7.05 & 0.00 & 1.00 & 74.77 & 17.18 \\
\hline Cocus nucifera & $\mathrm{PI}$ & Ex & 0.00 & 11.11 & 0.00 & 0.00 & 0.00 & 0.00 & 1.85 \\
\hline Dypsis lutescens & $\mathrm{PI}$ & Ex & 17.90 & 0.00 & 6.27 & 0.00 & 6.00 & 0.00 & 5.03 \\
\hline Elaeis oleifera & $\mathrm{PI}$ & $\mathrm{Nt}$ & 0.00 & 4.17 & 48.56 & 4.66 & 24.99 & 2.88 & 14.21 \\
\hline Livistona chinensis & $\mathrm{PI}$ & Ex & 5.01 & 27.78 & 0.00 & 17.10 & 0.00 & 0.00 & 8.32 \\
\hline Mauritia flexuosa & $\mathrm{PI}$ & $\mathrm{Nt}$ & 0.72 & 8.33 & 2.35 & 0.00 & 0.00 & 0.00 & 1.90 \\
\hline \multicolumn{10}{|l|}{ Bignoniaceae } \\
\hline Handroanthus sp. & $\mathrm{Ar}$ & Ind & 0.00 & 0.00 & 0.00 & 1.55 & 0.00 & 0.00 & 0.26 \\
\hline \multicolumn{10}{|l|}{ Chrysobalanaceae } \\
\hline Couepia subcordata & $\mathrm{Ar}$ & $\mathrm{Nt}$ & 7.87 & 1.39 & 0.00 & 0.00 & 0.00 & 0.00 & 1.54 \\
\hline \multicolumn{10}{|l|}{ Clusiaceae } \\
\hline Garcinia madruno & $\mathrm{Ar}$ & $\mathrm{Nt}$ & 0.72 & 0.00 & 0.00 & 0.00 & 0.00 & 0.00 & 0.12 \\
\hline \multicolumn{10}{|l|}{ Combretaceae } \\
\hline Terminalia catappa & $\mathrm{Ar}$ & Ex & 0.00 & 1.39 & 3.13 & 0.00 & 10.00 & 0.00 & 2.42 \\
\hline \multicolumn{10}{|l|}{ Fabaceae } \\
\hline Acacia sp. & $\mathrm{Ar}$ & Ind & 0.72 & 0.00 & 0.00 & 0.00 & 0.00 & 0.00 & 0.12 \\
\hline Caesalpinia sp. & $\operatorname{Ar}$ & Ind & 0.00 & 2.78 & 0.00 & 0.00 & 0.00 & 0.00 & 0.46 \\
\hline Cassia fistula & $\mathrm{Ar}$ & Ex & 0.72 & 0.00 & 0.00 & 0.00 & 0.00 & 0.00 & 0.12 \\
\hline \multicolumn{10}{|l|}{ Moraceae } \\
\hline Ficus benjamina & $A b$ & Ex & 10.74 & 5.56 & 12.53 & 10.88 & 1.00 & 0.00 & 6.78 \\
\hline \multicolumn{10}{|l|}{ Myrtaceae } \\
\hline Syzygium cumini & $\mathrm{Ar}$ & Ex & 0.00 & 0.00 & 0.00 & 24.87 & 8.00 & 0.00 & 5.48 \\
\hline Syzygium malaccense & $\mathrm{Ar}$ & Ex & 5.01 & 4.17 & 1.57 & 3.11 & 0.00 & 0.00 & 2.31 \\
\hline \multicolumn{10}{|l|}{ Sapotaceae } \\
\hline Pouteria caimito & $\mathrm{Ar}$ & $\mathrm{Nt}$ & 0.72 & 0.00 & 0.00 & 0.00 & 0.00 & 0.00 & 0.12 \\
\hline
\end{tabular}

RELACIÓN DE LA DIVERSIDAD DE AVES Y EL CLIMA, RUIDO YVEGETACIÓN

La variabilidad de las plazas con base en las aves, plantas, clima y ruido puede ser explicada en dos componentes o dimensiones al $60.55 \%$. En el componente I se puede explicar al $43.08 \%$, y las variables más importantes fueron las especies de plantas en general (-0.287), especies de plantas nativas $(-0.280)$, especies de árboles $(-0.278)$, índice de abundancia de aves $(-0.269)$ y especies de aves carnívoras (-0.266). Es decir, donde hay más especies de plantas se tiene más abundancia de aves, sobre todo de especies de aves carnívoras. Las plazas con estas características fueron: 28 de Julio, Armas y Sargento Lores.

El componente II puede explicar la variabilidad al $18.08 \%$ y las variables más importantes fueron la temperatura del suelo (-0.397) y las especies de aves frugívoras-insectívoras (0.327) (Figura 5). 

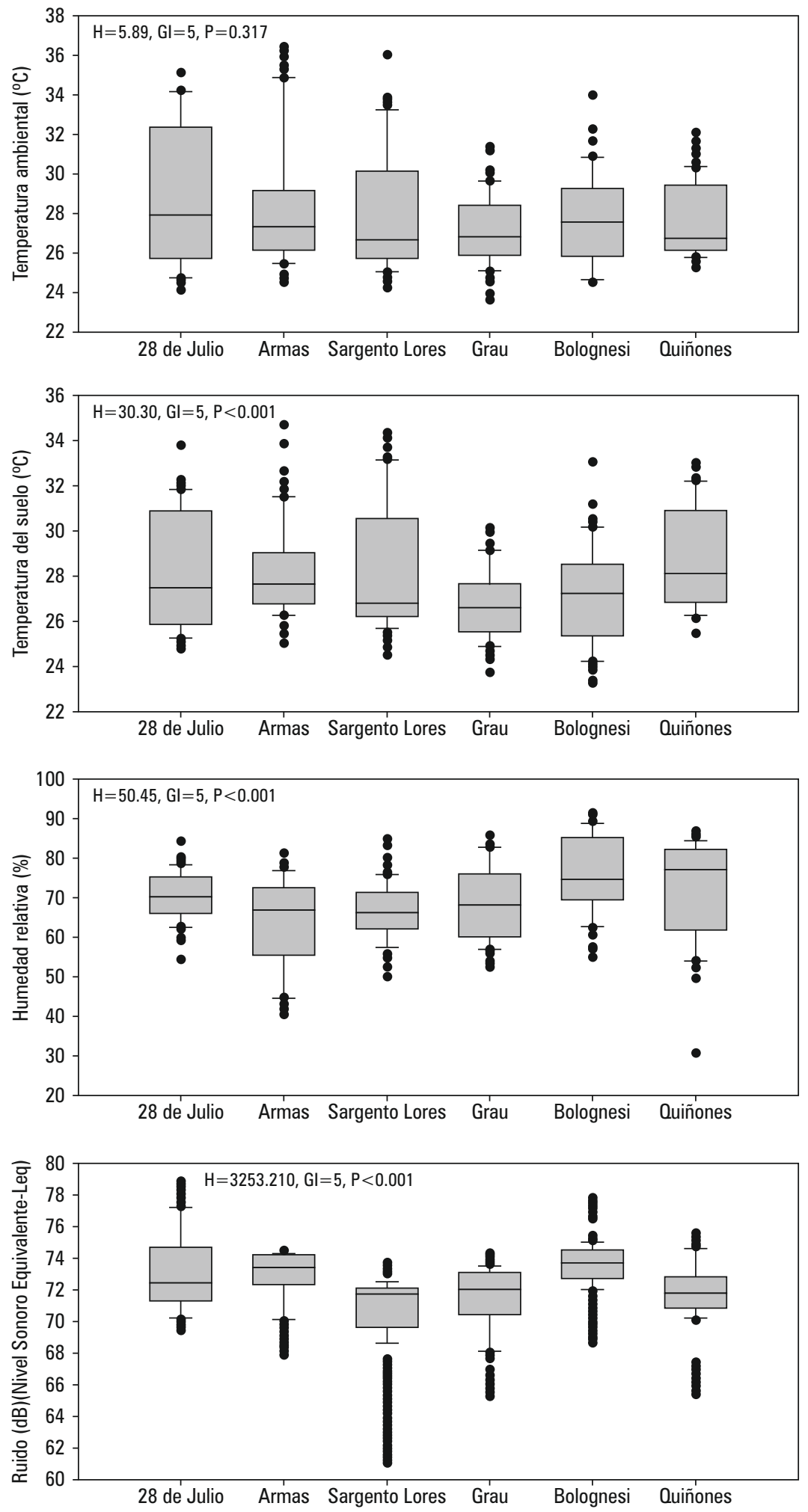

Figura 4. Temperatura ambiental $\left({ }^{\circ} \mathrm{C}\right)$, temperatura del suelo $\left({ }^{\circ} \mathrm{C}\right)$, humedad relativa $(\%)$ y ruido $(\mathrm{dB})$ (Nivel Sonoro Equivalente - Leq) registradas en las plazas de la ciudad de lquitos. 
Tabla 4. Prueba post-hoc utilizando Turkey para compara la temperatura del suelo y humedad entre las plazas, y la prueba de Dunn, para comparar el ruido en las plazas de Iquitos. Las palabras indican si hubo diferencias o no entre pares de plazas.

\begin{tabular}{|c|c|c|c|c|c|c|c|}
\hline Variables & Plazas & 28 de Julio & Armas & $\begin{array}{c}\text { Sargento } \\
\text { Lores }\end{array}$ & Grau & Bolognesi & Quiñones \\
\hline \multirow{5}{*}{$\begin{array}{l}\text { Temperatura } \\
\text { del suelo }\left({ }^{\circ} \mathrm{C}\right)\end{array}$} & 28 de Julio & & NO & NO & SI & NO & NO \\
\hline & Armas & & & NO & SI & NO & NO \\
\hline & Sargento Lores & & & & NO & NO & NO \\
\hline & Grau & & & & & NO & SI \\
\hline & Bolognesi & & & & & & $\mathrm{SI}$ \\
\hline \multirow{5}{*}{ Humedad (\%) } & 28 de Julio & & SI & NO & NO & NO & NO \\
\hline & Armas & & & NO & NO & SI & SI \\
\hline & Sargento Lores & & & & NO & SI & SI \\
\hline & Grau & & & & & $\mathrm{SI}$ & NO \\
\hline & Bolognesi & & & & & & NO \\
\hline \multirow{5}{*}{ Ruido (dB) } & 28 de Julio & & SI & SI & SI & SI & SI \\
\hline & Armas & & & SI & SI & SI & SI \\
\hline & Sargento Lores & & & & SI & $\mathrm{SI}$ & $\mathrm{SI}$ \\
\hline & Grau & & & & & SI & SI \\
\hline & Bolognesi & & & & & & SI \\
\hline
\end{tabular}

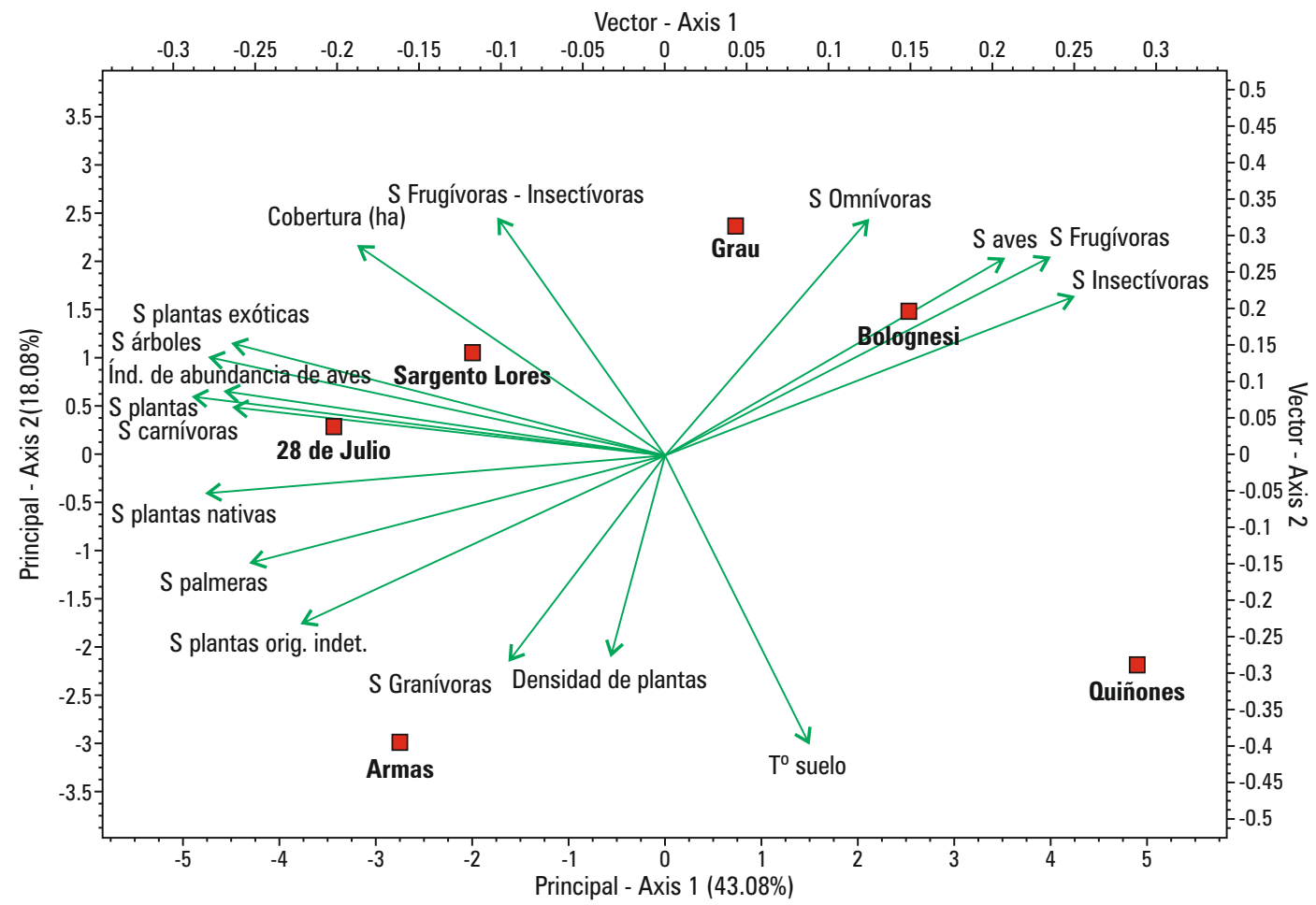

Figura 5. Análisis de componentes principales para determinar las variables que afectan la diversidad de aves en las plazas de la ciudad de lquitos. 
El Grupo I conformado por la Plaza 28 de Julio, Armas y Sargento Lores y el Grupo II conformado por la Plaza Grau, Bolognesi y Quiñones presentaron diferencias significativas (ANOSIM, $\mathrm{P}=0.05$ ), debido a que el Grupo II posee un mayor número de especies de aves omnívoras, especies de aves frugívoras y mayor temperatura del suelo.

\section{DISCUSIÓN}

En el presente estudio se registró un total de 50 especies de aves agrupadas en 12 órdenes y 20 familias, ligeramente menor al estudio de diversidad de aves en ambientes urbanos de la ciudad de Iquitos, donde se registró 56 especies de aves pertenecientes a 12 órdenes y 23 familias (Orbe \& Quispe 2015), quizá debido al mayor área de muestreo, incluyendo más calles provistas de más especies de plantas que sirven como alimento, dormideros, lugares de reproducción y nidificación, los cuales permiten que muchas especies de aves sean residentes. Asimismo, las diferencias quizá fueron debido al horario, horas de esfuerzo y la temporada de muestreo.

Las especies más abundantes según el índice de abundancia fueron Columba livia (31.36 ind./hora), Coragyps atratus (23.14ind./hora) y Tyrannus savana (5.79 ind./hora). Según lo reportado por Orbe y Quispe (2015), las especies más abundantes fueron similares, caso de Columbia livia $(34.16 \%)$, Brotogeris versicolurus (14.6\%) y Coragyps atratus (14.41\%). Columba livia domina el ambiente urbano, en donde encuentra las condiciones favorables de reproducción y refugio, resultando el incremento de su población. En adición, la especie no encuentra predadores naturales como las rapaces, y por su baja exigencia alimentaria tiene alta disponibilidad de alimento (Martins et al., 2015). Coragyps atratus, es una especie con amplia distribución y tolerante a altos grados de perturbación, y se beneficia de los desechos del hombre, además las infraestructuras antrópicas proporcionan refugio (Ruíz, 2014).Tyrannus savana que fue reportada como especie dominante en el presente estudio, no fue registrado por Orbe \& Quispe (2015), debido a que esta especie es migrante austral, y puede ser encontrada en bandadas grandes en los meses de febrero a abril y de agosto a octubre (Schulenberg et al., 2010).

Los registros de las especies de hábitos acuáticos y ribereños como Ardea alba, Butorides striata, Phalacrocorax brasilianus, Egretta thula, Megaceryle torquata y Phaetusa simplex en las plazas durante los días de muestreos, indican que estas especies estuvieron de paso hacia las orillas del río Itaya, Nanay o lago de Moronacocha, los cuerpos de aguas más cercanos a la ciudad de Iquitos.
Las plazas tuvieron mayor densidad de Adonidia merrillii, Elaeis oleifera, Livistona chinensis y Ficus benjamina, debido posiblemente a la política de gestión de las municipalidades, que son las encargadas de determinar las especies y cantidades a sembrar en las calles, parques o plazas de la ciudad. Desde un vivero se abastece de plantas ornamentales a las principales municipalidades, sobre todo de aquellas especies de plantas ornamentales que han sido introducidas por las autoridades municipales desde hace años y desde varios lugares (Zárate \& Mori, 2012), por esta razón dominan las plantas ornamentales exóticas, dejando de lado a muchas especies de plantas nativas de uso ornamental. Además, otro factor que puede estar favoreciendo la alta densidad de estas plantas en las plazas de Iquitos es su fácil propagación, que usualmente se realiza por medio de esquejes, donde posteriormente las plántulas son llevadas a los diversos distritos que lo requieren (Zárate \& Mori, 2012).

No hubo diferencia en la temperatura ambiental porque el aire homogeniza la temperatura en las plazas estudiadas. No obstante, hay mayor temperatura en el interior de la ciudad que la periferia (Ángel et al., 2010). Se asume que, la vegetación de orillas contribuye a la reducción de la temperatura, mediante la reducción o control de la radiación solar (Moreno, 2010).

También se asume que, la diferencia existente en la temperatura del suelo entre las plazas sea debida a la influencia de la vegetación sobre la temperatura del suelo. Las plazas difieren en cobertura vegetal, por ello el suelo sin vegetación rápidamente incrementa su temperatura de superficie (Chiarito \& Chiarito, 2015). Es por esta razón es que la Plaza Quiñones, al poseer menor cobertura de vegetación, posee una alta temperatura del suelo; por otro lado, la Plaza Grau es la que posee una mayor cobertura de plantas y por tanto posee la menor temperatura del suelo.

Los valores de ruido difieren entre las plazas debido a las diferencias de tránsito, algunas están en zonas más comerciales y otras en calles con alto tránsito. Las Plaza Bolognesi, Plaza de Armas y Plaza 28 de Julio, poseen los mayores valores de ruido debido a que están ubicadas en zonas céntricas de la ciudad, rodeadas por vías importantes con gran ruido. Este es el caso del cruce de las avenidas Grau, Abelardo Quiñones y Participación, en donde se encuentra la Plaza Bolognesi, así como el cruce entre los Jirones Putumayo, Arica y Próspero, donde se encuentra la Plaza de Armas; además el cruce entre los Jirones Tacna y Av. Mariscal Cáceres, en donde está la Plaza 28 de Julio (Digesa, 2008; Gratelly et al. 2012). 


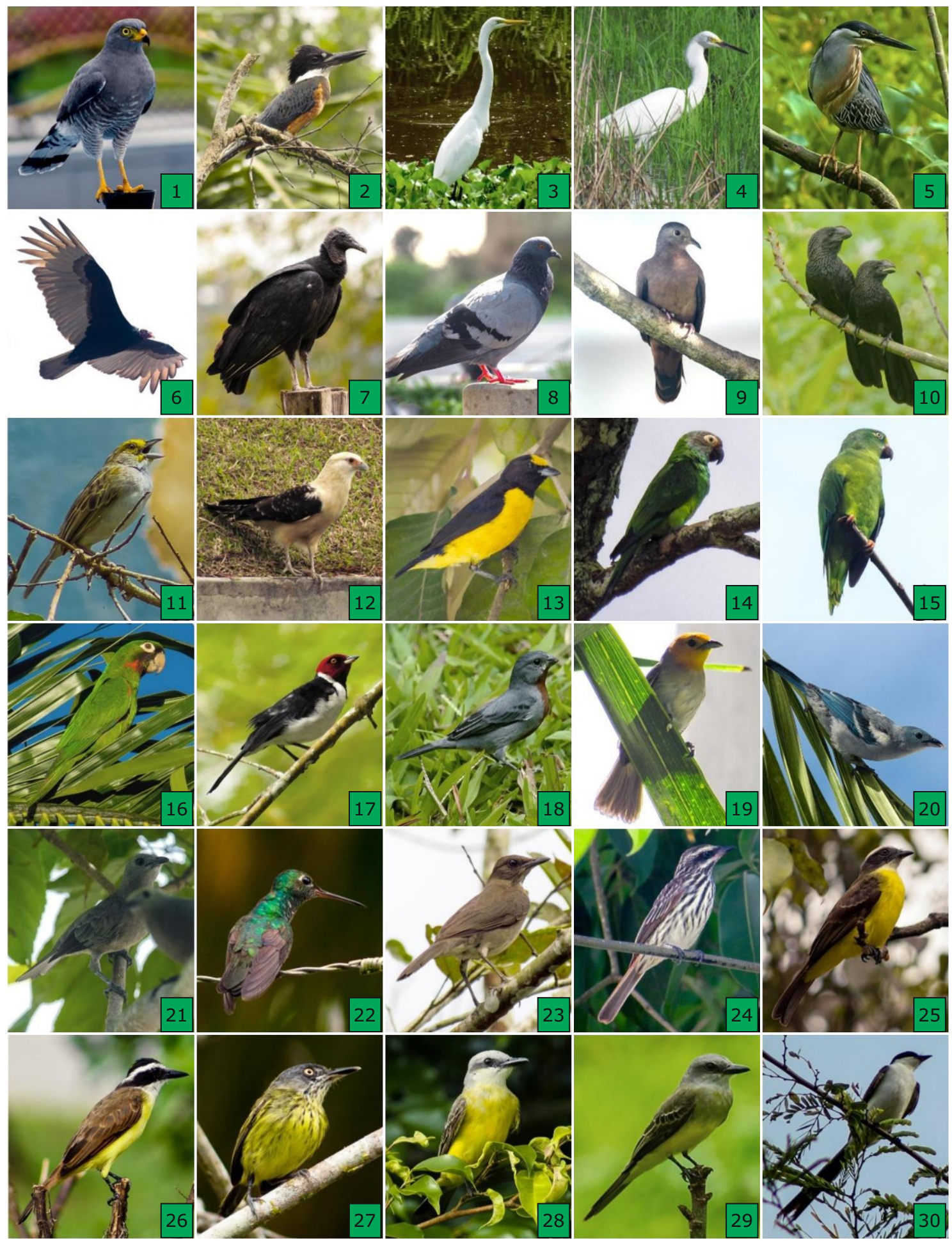

Principales aves de la ciudad de Iquitos. 1. Rupornis magnirostris, 2. Megaceryle torquata, 3. Ardea alba, 4. Egretta thula, 5. Butorides striata, 6. Cathartes aura, 7. Coragyps atratus, 8. Columba livia, 9. Columbina talpacoti, 10. Crotophaga ani, 11. Ammodramus aurifrons, 12. Milvago chimachima, 13. Euphonia chlorotica, 14. Aratinga weddellii, 15. Brotogeris sancticthomae, 16. Psittacara leucophthalmus, 17. Paroaria gularis, 18. Sporophila castaneiventris, 19. Tlypopsis sordida, 20. Thraupis episcopus, 21. Thraupis palmarum, 22. Amazilia fimbriata, 23. Turdus ignobilis, 24. Myiodynastes maculatus, 25. Myiozetetes similis, 26. Pitangus sulphuratus, 27. Todirostrum maculatum, 28. Tyrannus albogularis, 29. Tyrannus melancholicus, 30. Tyrannus savana. Fotografías de José Alonso Armas Silva. 
La Plaza Quiñones y la Plaza Grau poseen los valores medios de ruido, debido a que están cercanas solo a una vía considerada como ruidosa, que es la Avenida Abelardo Quiñones, con un valor promedio de 71.95 LAeqT y la Av. La Marina con 28 de Julio con 75.53 LAeqT para el caso de la Plaza Grau (Silva, 2016). En el caso de la Plaza Sargento Lores, esta posee el valor más bajo de ruido debido a que no se encuentra ubicada cerca de una vía con alto flujo vehicular, puesto que ninguna de las calles que lo rodean son consideradas como punto con gran ruido.

El estudio determinó la relación entre la abundancia de aves, específicamente entre el número de especies de aves carnívoras, con el número de especies de plantas, plantas nativas $\mathrm{y}$ árboles. La riqueza y diversidad de aves suelen ser relativamente más altas en presencia de especies vegetales nativas (Rivera-Gutiérrez, 2006). De igual forma, la presencia de plantas, sobre todo la presencia de árboles altos, proporcionaría mayor oferta de alimento, sitios de perchado y de nidificación, para garantizar una sobrevivencia de las especies carnívoras (DeGraaf, 1991). Este es un buen indicador para aumentar considerablemente la cantidad de especies de árboles, sobre todo de especies nativas en las plazas locales.

Las aves carnívoras, como las reportadas en el presente estudio, están compuestas básicamente por aves rapaces, las mismas que son depredadores de alto nivel trófico que determinan la estructura y organización de las comunidades biológicas (Rau, 2014). Este grupo de aves, es particularmente sensible a la modificación o pérdida de sus ambientes naturales causados por actividades humanas o fenómenos naturales, por lo que son consideradas un excelente indicador biológico, cuya ausencia puede significar grandes cambios en los lugares donde suelen habitar (Márquez et al., 2005; Vázquez-Pérez et al., 2009; Medina et al. 2012). Es decir, con su presencia pueden indicar el buen estado de conservación de un bosque.

Se considera que, a un mayor número de especies carnívoras mejor será la condición de las plazas en la ciudad de Iquitos, y por consiguiente mejores condiciones de la ciudad. Además, al estar relacionadas las aves carnívoras con las especies de plantas, especies de plantas exóticas y especies de árboles, se puede decir que estas aves son indicadoras de una ciudad verde. Es decir, una ciudad con bosque en su interior, es ideal para numerosas especies de animales que habitan en la ciudad, incluyendo al hombre. En consecuencia, estas aves carnívoras juegan un papel clave en nuestros ecosistemas, y que, al mantener sus poblaciones, mantendrán la salud de estos ecosistemas.
Asimismo, se determinó que existe una relación inversamente proporcional entre la temperatura del suelo y las especies de aves frugívoras-insectívoras. La temperatura del suelo es un factor iniciador y regulador de los procesos de metabolismo, crecimiento y desarrollo de la planta, pues algunos órganos y hasta la propia planta puede morir cuando la temperatura rebasa los límites de un rango determinado (Fisher et al., 1997); aunque la producción de frutos se ve incrementada por el aumento de la temperatura del suelo $\left(25-35^{\circ} \mathrm{C}\right)$ (Fisher et al., 1997), esta puede disminuir cuando las temperaturas son muy elevadas. La temperatura del suelo también determina los ciclos de vida de insectos y otros invertebrados que salen del suelo dependiendo su temperatura (GLOBE, 2005), aunque las altas temperaturas del suelo pueden ser letales para los insectos, hongos, nemátodos y bacterias en el suelo (Cañedo et al., 2011), donde para el caso de los insectos, la temperatura del suelo letal es mayor a $45^{\circ} \mathrm{C}$ (Pruett \& Guamán, 2001). Los altos niveles de temperatura en el suelo reducen la producción de frutos y la presencia de insectos en el suelo, por lo que la presencia de especies de aves frugívoras-insectívoras también se verá reducida al no tener una alta disponibilidad de alimento.

\section{CONCLUSIÓN}

La biodiversidad y abundancia de aves carnívoras, está estrechamente relacionada con las plantas, específicamente con el número de especies de plantas en general, especies de plantas nativas y especies de árboles. Esto indica que las especies carnívoras o rapaces pueden ser indicadoras de la conversión hacia una ciudad verde, un ambiente que mejora la calidad de vida de las sociedades humanas.

\section{AGRADECIMIENTOS}

A YAVACUS por facilitar la ejecución de este estudio. Al IIAP por habernos brindado los materiales y equipos necesarios para la colecta de plantas. A Percy Saboya Del Castillo por su ayuda en la identificación de las aves. A Marcos Ríos del IIAP y Chi Yung Jim del Departamento de Geografía de la Universidad de Hong Kong, por la identificación de plantas. A la Municipalidad Provincial de Maynas por el permiso otorgado en la colecta de plantas. Al SERFOR del Ministerio de Agricultura por el permiso de investigación otorgado $\left(\mathrm{N}^{\circ} 0068\right.$-2015-SERFOR-DGGSPFFS $)$ que hizo posible la realización de este estudio. A nuestros amigos, Alexis Aching y Oscar Alcántara por habernos apoyado en la medición del ruido en las plazas de Iquitos. 


\section{BIBLIOGRAFÍA}

Álvarez, J.; Díaz, J.; Shany, N. 2012. Avifauna de la Reserva Nacional Allpahuayo Mishana. Cotinga, 34: 61-84.

Ángel, L.; Ramírez, A.; Domínguez, E. 2010. Islas de color y cambios espacio-temporales de la temperatura en la ciudad de Bogotá. Revista de la Real Academia Colombiana de Ciencias Exactas, Físicas y Naturales, 34 (131): 173-183.

APG III (Angiosperm Phylogeny Group). 2009. An update of the Angiosperm Phylogeny Group classification for the orders and families of flowering plants. Botanical Journal of the Linnean Society, 161 (2): 105-121.

Castañeda, L.Z.; Arnao, L.M.; Castillo, L.N.; Álvarez, S.C.; Quinteros, Z.D.; Caro, C.C. 2013. PERÚ. En: Ecología urbana: Experiencias en América Latina. Ortega-Álvarez, R (Eds). 1a ed. MacGregor-Fors, I. México. p.100-110.

Bazán, G.L. 2012. Riqueza, Abundancia y Diversidad de Aves en el Área de Conservación Municipal "Bosque de Huamantanga" Jaén, Perú. Tesis de pre-grado, Universidad Nacional de Trujillo, Facultad de Ciencias Biológicas, Trujillo, Perú. 75pp.

Berget, C. 2006. Efecto del tamaño y de la cobertura vegetal de parques urbanos en la riqueza y diversidad de la avifauna de Bogotá, Colombia. Gestión y Ambiente, 9 (2): 45-60.

Cañedo, V.; Alfaro, A.; Kroschel, J. 2011. Manejo integrado de plagas de insectos en hortalizas. Principios y referencias técnicas para la Sierra Central de Perú. Centro Internacional de la papa (CIP). Lima, Perú. 48pp.

Cárdenas, D.; Castaño, N.; Cárdenas-Toro, J. 2011. Plantas introducidas, establecidas e invasoras en Amazonía Colombiana. Instituto Amazónico de Investigaciones Científicas - Sinchi. Bogotá, Colombia. 153pp.

Castillo, L.; Castañeda, L.; Quinteros, Z. 2014. Aves del campus de la Universidad Nacional Agraria La Molina (Lima-Perú) - Una revisión de su abundancia, distribución y diversidad desde 1992 al 2010. Ecología Aplicada, 13 (2): 117128.

Castro, A. 2012. Caracterización del potencial aviturístico en un paisaje rural en los Llanos Orientales. Puerto López-Meta. Tesis de pregrado, Pontificia Universidad Javeriana, Facultad de Estudios Ambientales y Rurales, Bogotá. 89pp.

Castro, F.; Gonzales, M. 2014. Caracterización Biológica de La Reserva Bojonawi (Puerto Carreño, Vichada). Documento Técnico.
Fundación Palmarito y Fondo para la Acción Ambiental y la Niñez. 165pp.

Chiarito, G; Chiarito, E. 2015. Evaluación de temperaturas urbanas de acuerdo al uso del suelo: Rosario. Avances en Energías Renovables y Medio Ambiente, 19: 01.93-01.102.

Crocce, I. 2011. Comunidad de aves y peligro aviario en el Aeropuerto Internacional de Carrasco. Tesis de pre-grado, Universidad de la República, Facultad de Ciencias, Uruguay. 34pp.

DeGraaf, R.M. 1991. Winter foraging guild structure and habitat associations in suburban bird communities. Landscape and Urban Planning, 21: 173-180.

Dirección General de Salud Ambiental (DIGESA). 2008. Estudio de la calidad del aire en la Ciudad de Iquitos, Provincia de Maynas. Documento técnico. Ministerio de Salud. 16pp.

Dourojeanni, M. 2013. Loreto sostenible al 2021. Derecho, Ambiente y Recursos Naturales. Primera Edición. Perú. 356pp.

Fisher, G.; Torres, F.; Torres, J. 1997. Efecto de la temperatura del suelo sobre la planta. Revista Comalfi, 14 (3): 78-92.

Global Learning and Observations to Benefit the Environment (GLOBE). 2005. Protocolo de Temperatura del suelo. $17 \mathrm{pp}$.

Gratelly, P.A.; Oliveira, L.F.; Ikeda, S.B. 2012. Valoración económica de la contaminación sonora del parque automotor de Iquitos, Loreto. Conocimiento amazónico, 3 (2): 131-138.

Judd, W., Campbell, C., Kellogg, E.; Stevens, P. 1999. Plant Systematics A phylogenetic approach. Sinauer Associates, Inc. Sunderland, Reino Unido. 464pp.

Kardous, C.; Shaw, P. 2014. Evaluation of smartphone sound measurement applications. The Journal of the Acoustical Society of America, 135 (4): 186-192.

Londoño-Betancourth, J.C. 2013. Discusiones sobre la presencia de aves rapaces, aves migratorias y aves bajo algún grado de amenaza en la ciudad de Pereira, Risaralda. Luna Azul, 36: 134-164.

Marengo, J.A. 1998. Climatología de la zona de Iquitos, Perú. En: Geoecología y desarrollo amazónico, estudio integrado en la zona de Iquitos, Perú. Kalliola, R.; Flores, P.S. (Eds). Turku (Finlandia): Turunyliopisto. 544 pp.

Márquez, C.; Bechard, M.; Gast, F.; Vanegas, VH. 2005. Aves rapaces diurnas de Colombia. Instituto de Investigación de Recursos Biológicos "Alexander von Humboldt". Bogotá, Colombia. 394 pp. 
Martins, C.M.; Welkel, A.; Francini, K.; Tostes, S. 2015. Percepção de usuários de espaços públicos de Curitiba, Paraná, sobre a presença de pombos (Columba livia). Archives of Veterinary Science, 20 (4): 10-19.

Medina, S.M.; Rojo, G.E.; Martínez, R.; Piña, H.H.; Lara, E. 2012. Abundancia, diversidad y uso de hábitat de aves rapaces diurnas en el norte de Sinaloa. En: Recursos naturales y contaminación ambiental. Primera Edición. México. p. 99-115.

Moreno, M.C. 2010. Climatología urbana. Universidad de Barcelona, España. 75pp.

Navarro-Sigüenza, A.G.; Rebón-Gallardo, M.F.; Gordillo-Martínez, A.; Townsend, A.; BerlangaGarcía, H-; Sánchez-González, L.A. 2014. Biodiversidad de aves en México. Revista Mexicana de Biodiversidad, 85: 476-495.

Nolazco, S. 2012. Diversidad de aves silvestres y correlaciones con la cobertura vegetal en parques y jardines de la ciudad de Lima. Boletín informativo UNOP, 7 (1): 4-16.

Ochoa, B.; Pasquali, C. 2010. Ecoturismo urbano: ¿Existen condiciones para desarrollar la observación de aves, como actividad turística en Caracas?. Multiciencias, 10 (2): 117-124.

Orbe, M.P.; Quispe, L.M. 2015. Diversidad de aves en ambientes urbanos y periurbanos de la ciudad de Iquitos y bosque de varillal, Loreto - Perú. Tesis de pre-grado, Universidad Nacional de la Amazonía Peruana, Facultad de Ciencias Biológicas, Iquitos, Perú. 84pp.

Peña-Ñúnez, J.L.; Claros-Morales, A.F. 2016. Estudio preliminar de la avifauna en el campus de la Universidad de la Amazonia, en Florencia, Caquetá, Colombia. Revista de Biodiversidad Neotropical, 6 (1): 85-92.

Plenge, MA. 2017. Lista de las Aves de Perú. Boletín informativo UNOP. $41 \mathrm{pp}$.

Pruett, C.; Guamán, I. Principio de manejo integrado de plagas y biocontrol en siembra directa. En: Siembra Directa en el Cono Sur. Díaz, R. (coord.). Montevideo, Uruguay. p. 121-157.

Rau, J. 2014. Papel ecológico de las aves rapaces: del mito a su conocimiento y conservación en Chile. Departamento de Ciencias Biológicas y Biodiversidad, Universidad de Los Lagos, Campus Osorno, Chile. 38pp.

Rivera-Gutiérrez, HF. 2006. Composición y estructura de una comunidad de aves en un área suburbana en el suroccidente colombiano. Ornitología colombiana, (4): 28-38.

Romero, F. 2015. Relación entre el ruido ambiental e influencia de su componente frecuencial con la diversidad de avifauna en parques y jardines.
Tesis de post-grado, Universidad de Extremadura, Departamento de Biología Vegetal, Ecología y Ciencias de la Tierra, España y Portugal. 232pp.

Ruíz, C. 2014. Bioecología del Coragyps atratus "Gallinazo" en la zona del distrito de Belén Perú. Tesis de pre-grado, Universidad Nacional de la Amazonía Peruana, Facultad de Agronomía, Iquitos, Perú. 80pp.

Salazar, E.; Mattos, J.; Díaz, J.; Ferreyra, F.; Piana, R.; Balta, K. 2003. Composición de especies de aves en hábitats de la zona reservada Allpahuayo - Mishana y colinas de la formación Nauta, Loreto, Perú. Folia Amazónica, 14 (1): 125-132.

Schulenberg, T.; Stotz, D.; Lane, D.; O'Neill, J.; Parker III, T. 2010. Aves de Perú. Serie en Biodiversidad Corbidi 01. CORBIDI. Lima, Perú. 460 pp.

Silva, F.A. 2016. Evaluación de los niveles de ruido en zonas de las Avenidas La Marina y Abelardo Quiñones de la Ciudad de Iquitos, Loreto, 2014. Tesis de pre-grado, Universidad Nacional de la Amazonía Peruana, Facultad de Agronomía, Iquitos, Perú. 71pp.

Siqueira, P.R.; Ferreira, M.; Gonçalves, R.M., Leite, L.O. 2015. Assessment of stomach contents of some amazonian birds. Ornitología Neotropical, 26: 79-88.

Strewe, R.; Villa-De León, C.; Alzate, J.; Beltrán, J.; Moya, J.; Navarro, C.; Utria, G. 2009. Las aves del Campus de la Universidad de Magdalena, Santa Marta, Colombia. Revista Intropica, 4: 79-91.

Takano, F.; Castro, N. 2007. Avifauna en el campus de la Universidad Nacional Agraria La Molina (UNALM), Lima - Perú. Ecología Aplicada, 6 (1-2): 149-154.

Vásquez, R. 1997. Flórula de las Reservas Biológicas de Iquitos, Perú. Missouri Botanical Garden Press. St. Louis-USA. 1046 pp.

Vázquez-Pérez, J.R.; Enríquez, P.L.; RangelSalazar, J.L. 2009. Diversidad de aves rapaces diurnas en la Reserva de la Biosfera Selva El Ocote, Chiapas, México. Revista Mexicana de Biodiversidad, 80:203-209.

Vásquez, R. 1997.Flórula de las Reservas Biológicas de Iquitos, Perú. Missouri Botanical Garden, 78:1002-1008.

Velásquez-Valencia, A.; Ricaurte, L.F.; Lara, F.; Cruz, E.J.; Tenorio, G.A., Correa M. 2003. Lista anotada de las aves de los humedales de la parte alta del Departamento de Caquetá. MEMORIAS: Manejo de Fauna Silvestre en Amazonía y Latinoamérica, 320-329. 
Velásquez, A. 2009. Estructura de la comunidad de aves en sistemas de producción del piedemonte amazónico. Informe final de post-grado, Universidad Nacional de Colombia, Facultad de Ciencias, Florencia, Caquetá. 51pp.

Zárate, R; Mori, T.J. 2012. Vegetación, documento temático. Proyecto Microzonificación Ecológica y Económica para el Desarrollo Sostenible del Área de Influencia de la Carretera Iquitos Nauta, convenio entre IIAP y DEVIDA. Iquitos, Perú. 257pp.
Haven, R.W.; Álvarez, J.; Díaz, J. 2015. Aves de Loreto bajo (60-250 m snm)/Birds of Lowland Loreto (60-250 m asl). Biology Department, University of North Carolina. 8pp.

Recibido: 10 de Julio del 2017

Aceptado para publicación: 28 de Julio del 2017 